\title{
Echocardiographic Guidance During Neonatal and Pediatric Jugular Cannulation for ECMO
}

\author{
Salazar, Paul A; Blitzer, David MD; Dolejs, Scott C MD MS; Parent, John J MD MSCR; \\ Gray, Brian W MD \\ Riley Hospital for Children, Indiana University School of Medicine
}

\begin{abstract}
Background: Internal jugular cannula position is traditionally confirmed via plain film at the conclusion of the peripheral ECMO cannulation procedure. However, it may be difficult to estimate the location of the right atrium on plain films. A misplaced cannula can result in need for repositioning and increased morbidity. Echocardiography (ECHO) may be used during cannulation as a more accurate means of guiding cannula position. The aim of this study is to study the effect of a protocol encouraging the routine use of $\mathrm{ECHO}$ at the time of cannulation.
\end{abstract}

Objective: To assess whether the use of echocardiographic guidance during neonatal and pediatric jugular cannulation for ECMO reduces the need for cannula repositioning or decreases morbidity.

Methods and Materials: We performed a retrospective review of patients at a single institution who received ECMO support using jugular venous cannulation from January 2013 through October 2016. We compared those who underwent $\mathrm{ECHO}(\mathrm{ECHO}+)$ at the time of cannulation with those who did not (ECHO-). Patient demographics and surgical history, ECMO cannulation details, ECMO events, need for cannula repositioning, cannula related morbidity, and patient outcomes were noted. The results were analyzed with descriptive and non-parametric statistics where applicable.

Results: 89 patients met inclusion criteria: $26 \mathrm{ECHO}+(29 \%), 63 \mathrm{ECHO}-(71 \%)$. Most of $\mathrm{ECHO}+$ patients underwent dual-lumen $\mathrm{VV}$ cannulation $(\mathrm{n}=17,65 \%)$, while $32 \%$ of ECHO- patients had VV support ( $\mathrm{p}<0.003)$. Seven $(27 \%) \mathrm{ECHO}+$ patients and $18(28 \%)$ ECHO- patients had a history of cardiac surgery prior to ECMO $(\mathrm{p}=0.88)$. All patients had CXR to verify cannula position, and fluoroscopy was used in $4 \mathrm{ECHO}+$ patients but no ECHO- patients. There was a major mechanical complication in each group: atrial perforation from a guidewire during cannulation in $\mathrm{ECHO}+$ and late atrial perforation from a loose cannula in ECHO-. Subsequent to cannulation, there were $0.58 \mathrm{ECHO}$ studies per patient to verify cannula position in the $\mathrm{ECHO}+$ group compared to $0.22 \mathrm{ECHO}$ per patient in the ECHO- group $(\mathrm{p}=0.02)$. Two $(8 \%) \mathrm{ECHO}+$ patients required a cannula repositioning procedure for misplacement during the ECMO run, while $6(10 \%) \mathrm{ECHO}$ - patients required repositioning procedures $(\mathrm{p}=0.78)$. In the $\mathrm{VV}$ ECMO subgroup, $\mathrm{ECHO}+$ patients required no repositioning, while $4(20 \%)$ of ECHO- VV patients required repositioning $(\mathrm{p}=0.1)$. Patients who had ECHO to verify cannula placement during the ECMO run were more likely to have a repositioning procedure $(\mathrm{p}<0.001)$. Repositioning procedures resulted in no additional complications. Survival to discharge was similar in both groups: $54 \%$ $\mathrm{ECHO}+$ and $62 \%$ ECHO- $(\mathrm{p}=0.51)$. 
Conclusion: Implementation of a protocol to perform ECHO during jugular cannulations for neonatal and pediatric peripheral ECMO did not result in significantly less repositioning procedures, complications, or ECHO studies performed per patient. ECHO may still be a useful adjunct for surgeons during difficult cannulations and for precise placement of a dual-lumen VV cannula, but this study does not support routine use.

Keywords: Respiratory failure, Circulatory failure, Pediatrics, Neonatal, Extracorporeal life support, ECMO, Veno-arterial, Veno-venous, Echocardiography,

\section{Introduction}

Extracorporeal membrane oxygenation (ECMO) enables cardiovascular and pulmonary recovery by providing prolonged cardiac and/or pulmonary bypass in the intensive care setting to severely ill patients with recoverable disease. ECMO cannulation involves the precise placement of a large catheter (cannula) within the central venous system for blood drainage from the patient to the ECMO circuit. More over, the majority of neonatal and pediatric ECMO support involves drainage of blood from the right atrium through the placement of a cannula within the right jugular vein with subsequent radiographic imaging to confirm cannula position.

Nevertheless, it is often difficult to estimate the location of the right atrium on plain films, and a misplaced cannula can result in ineffective ECMO support, significant morbidity to the patient, and excessive resource utilization to reposition the cannula. Blind cannulation can result in cardiac or vascular injury, and a cervical repositioning procedure while anticoagulated on ECMO can lead to morbidity from bleeding. ${ }^{1}$ As a result, recent literature has encouraged the use of echocardiography (ECHO) as a more accurate means of assessing cannula position by demonstrating that ECHO performed at the time of cannula placement reduces the need for cannula repositioning and may reduce morbidity associated with misplaced cannulae. ${ }^{2-4}$ Therefore, we set out to decipher the clinical and institutional effect after initiating a protocol encouraging the routine use of ECHO at the time of right internal jugular cannula placement in January 2015. Our primary aim was to determine if ECHO use decreased the need for cannula repositioning during the ECMO run. Our secondary aims were to evaluate for a change in cannula-related morbidity and resource utilization related to the ECHO protocol.

\section{Methods and Materials}

Following institutional review board approval (Indiana University IRB protocol \#1610913759), a retrospective review of patients at Riley Hospital for Children who received ECMO support using jugular venous cannulation from January 2013 through October 2016 was performed. We compared those who underwent $\mathrm{ECHO}(\mathrm{ECHO}+)$ at the time of cannulation with those who did not (ECHO-). 


\section{Cannulation Technique}

Cannulations were performed by a group of eight Pediatric General Surgeons in an Extracorporeal Life Support Organization ECMO center of excellence. All veno-arterial (VA) cannulations were through an open cervical cutdown technique. Ideal venous cannula tip placement was defined as mid right atrium with adequate ECMO flow to support the patient. The majority of veno-venous (VV) cannulations were through an open cervical cutdown technique, but there were some percutaneous cannulations. All VV cannulations were with the OriGen dual lumen cannula (OriGen Biomedical, Austin, Texas, USA). Ideal venous cannula tip placement was defined as low right atrium with adequate ECMO flow to support the patient.

\section{ECHO Technique}

Transthoracic echocardiography was performed under the sterile drapes at the time of ECMO cannulation with the patient in the supine position. The preferred imaging approach was subcostal with demonstration of the superior vena cava, right atrium, tricuspid valve, and right ventricle, typically utilizing a subcostal four-chamber view or bi-caval view. This allows for best visualization of the venous cannula to ensure it is past the right atrialsuperior vena cava junction, ideally in the low right atrium directed predominately toward the tricuspid valve (Fig 1A).

Furthermore, this view allows physicians to quickly assess the cannula to determine if it is undesirably deep (i.e. traverses the tricuspid valve or is into the right ventricular chamber) or if the cannula crosses a patent foramen ovale or atrial septal defect into the left atrium. In the event subcostal imaging quality is suboptimal or unobtainable, other views are utilized including apical four-chamber and parasternal long and short axis views. The addition of color Doppler was added to imaging to ensure proper venous inflow and outflow in the setting of veno-venous ECMO (Fig 1B). Cannula position was adjusted under ECHO guidance until the tip location was ideal and ECMO flow was optimal. 


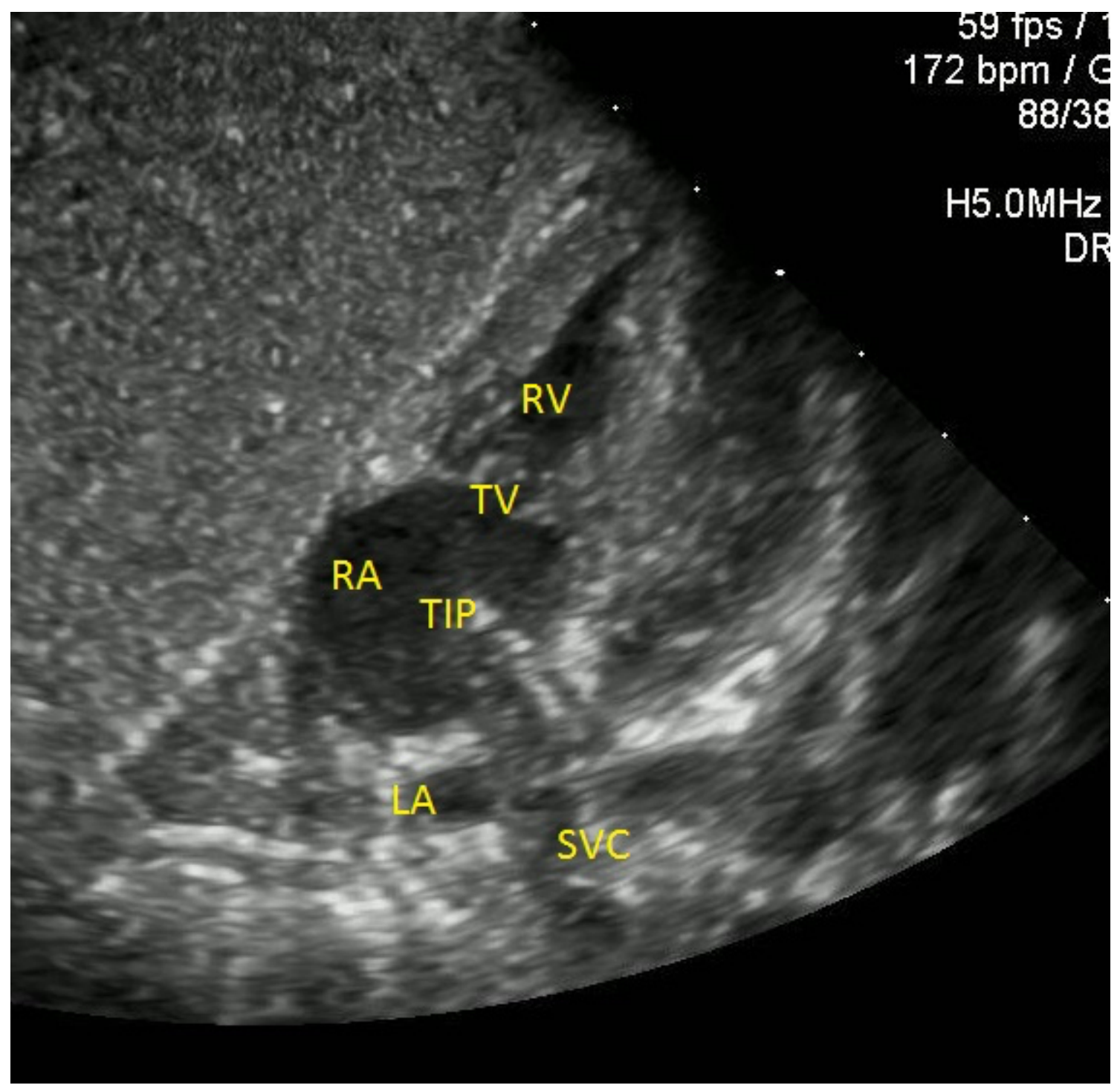

Figure $1 \mathrm{~A}$ : Echocardiography utilizing subcostal imaging window with demonstration of the superior vena cava (SVC), left atrium (LA), right atrium (RA), tricuspid valve (TV), right ventricle (RV) and venous cannula. Note tip of cannula in mid-lower right atrium. 


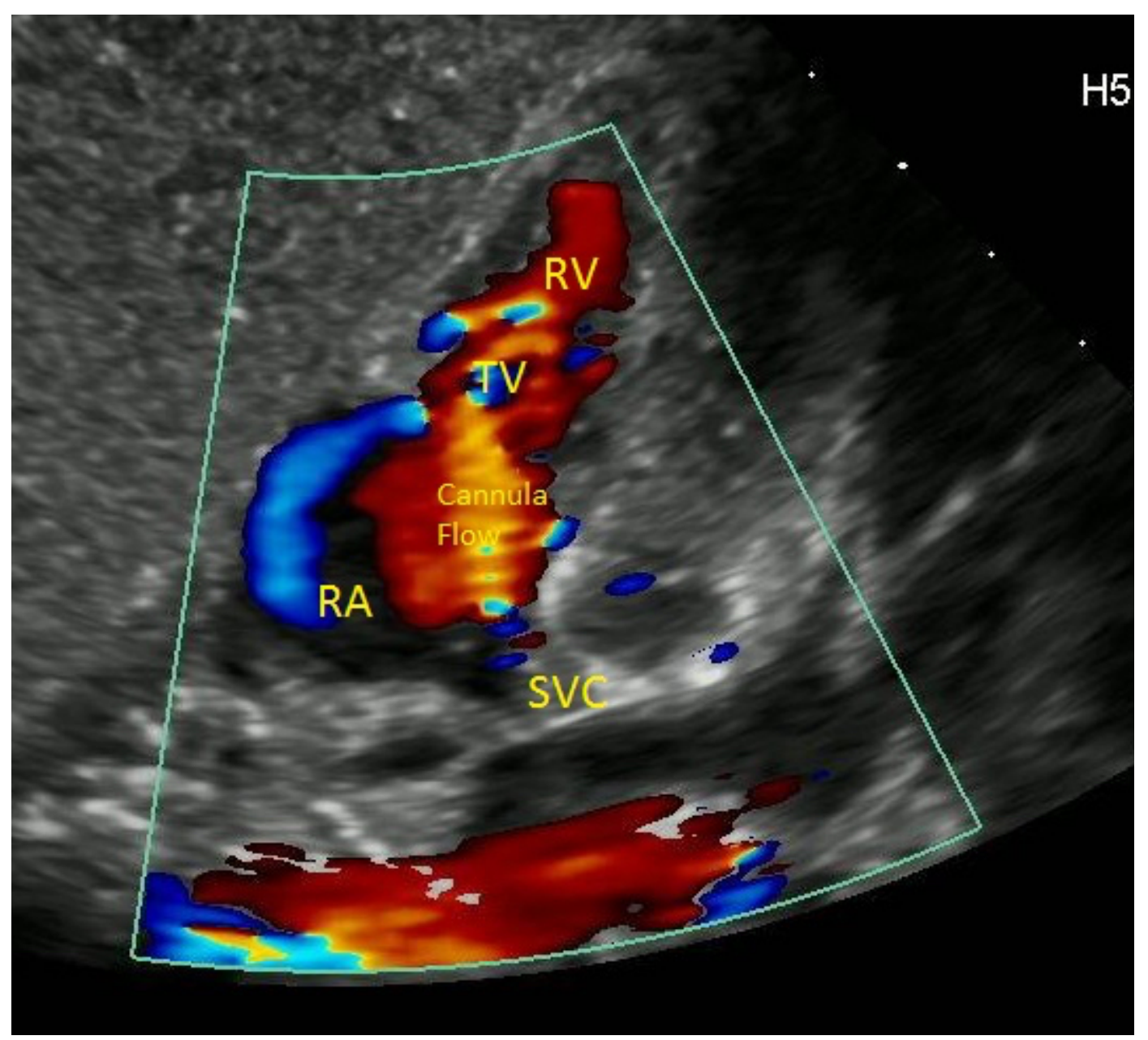

Figure 1B: Echocardiography in same imaging plane now with color Doppler showing venous cannula outflow (Red color labeled "Cannula Flow") directed toward the tricuspid valve as desired.

\section{Variables and Statistics}

We examined patient demographics and surgical history, ECMO cannulation details, ECMO events, need for cannula repositioning, cannula related morbidity, and overall patient outcomes. ECHO performed at time of cannulation is reported separately from subsequent ECHO studies performed during ECMO support to verify cannula placement. Any cannula repositioning procedure was a separate event from the original cannulation procedure and was at least 24 hours after initiation of ECMO.

For categorical variables, Fisher's exact or Chi-square tests were used to assess for significance, and significance was defined as $\mathrm{p}<0.05$. All statistical analysis was performed on SAS version 9.4 (Cary, NC).

\section{Results}




\section{Demographics and Survival}

There were 26 patients in the ECHO+ group and 63 in the ECHO- group. Table 1 shows the demographic breakdown of neonatal vs. pediatric patients in each group and ECMO diagnostic categories. The $\mathrm{ECHO}+$ group had a significantly higher proportion of pediatric patients as opposed to a higher proportion of neonates in ECHO-. There was no significant difference in diagnoses or survival to discharge between the groups. All patients had a chest $\mathrm{x}$-ray performed at the end of cannulation to verify cannula position. Fluoroscopy during cannulation was used in 4 ECHO+ patients, and no ECHO- patients underwent fluoroscopy.

\begin{tabular}{|c|c|c|c|}
\hline & ECHO (+) & ECHO (-) & P-Value \\
\hline N, Sample Size (\%) & $26(29)$ & $63(70)$ & --- \\
\hline Neonatal $(<1$ month $)$ & $4(15)$ & $35(56)$ & \multirow[b]{2}{*}{$<0.001$} \\
\hline Pediatric ( $>1$ month) & $22(85)$ & $28(44)$ & \\
\hline Cardiac & $8(31)$ & $23(37)$ & \multirow[b]{3}{*}{0.11} \\
\hline Respiratory & $18(69)$ & $32(51)$ & \\
\hline $\mathrm{CDH}$ & 0 & $8(12)$ & \\
\hline Survival to Discharge & $14(54)$ & $39(62)$ & 0.51 \\
\hline
\end{tabular}

Table 1. Demographics and Survival

\section{Modes of Cannulation}

Sixty-five percent of patients in the ECHO+ group were placed on VV ECMO support (17/26), while $35 \%$ of ECHO+ patients underwent VA ECMO support (9/26, Figure 2). Thirty-two percent of patients in the ECHO- group underwent VV ECMO (20/63), while $68 \%$ of ECHO- patients were supported with VA ECMO (43/63). This was statistically significant, with a p-value of 0.003 . 


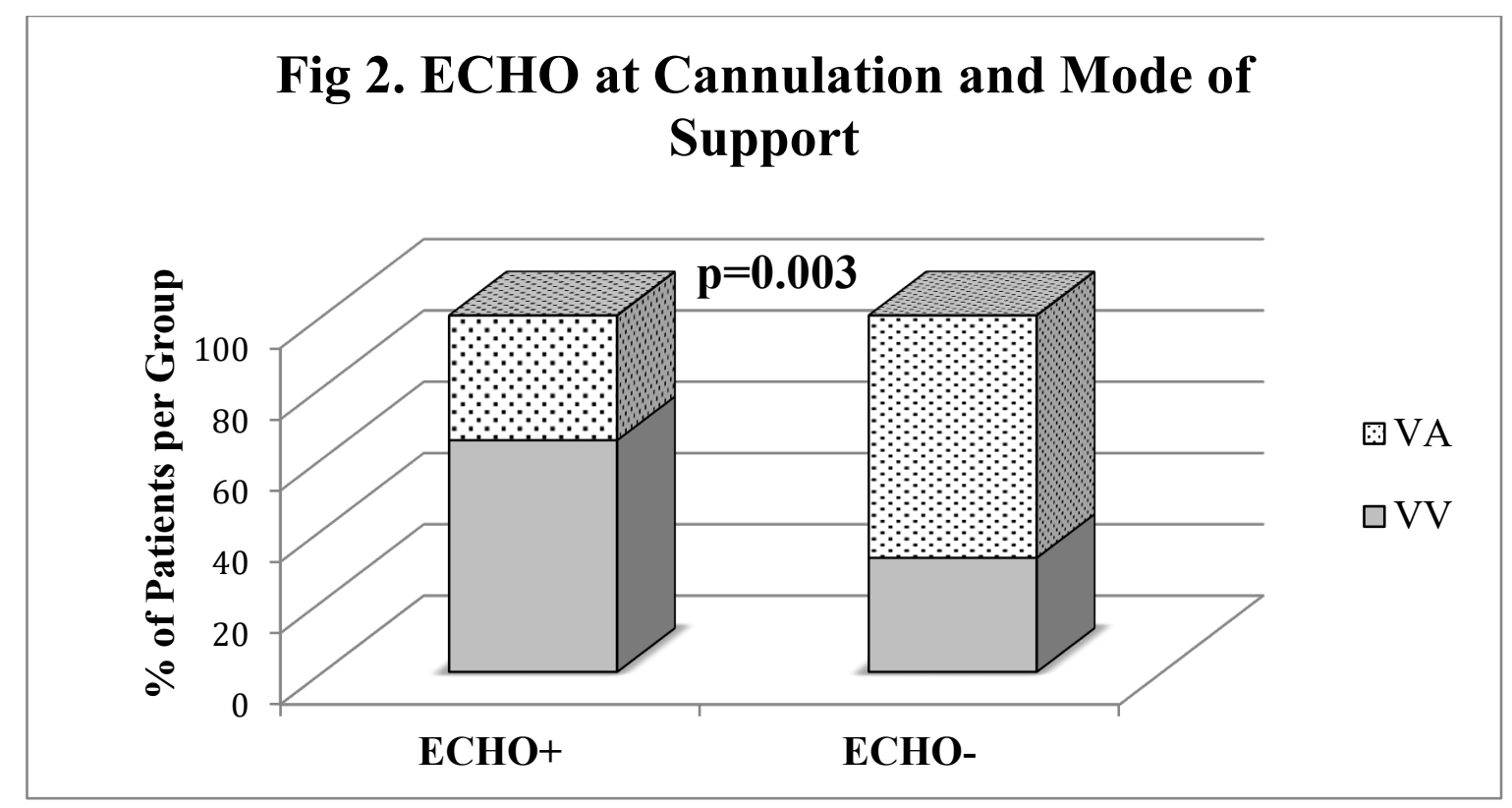

\section{Cannula Repositioning}

Eight patients required a jugular cannula repositioning procedure during their ECMO run after initial cannulation, and no patient had more than one repositioning procedure. There was no significant difference in repositioning when comparing the two groups: $2 / 26(8 \%)$ $\mathrm{ECHO}+$ vs. 6/63 (10\%) ECHO-, $\mathrm{p}=0.78$ (Figure 3). Patients who had an ECHO performed during their ECMO run to verify cannula position had greater likelihood of undergoing a cannula repositioning procedure: 7/20 (35\%) ECHO during ECMO vs. 1/69 (1.5\%), $\mathrm{p}<0.001$ (Figure 3). There were $0.58 \mathrm{ECHO}$ studies per patient performed to verify cannula position in the ECHO+ group, compared to 0.22 ECHO studies per patient in the ECHOgroup $(\mathrm{p}=0.02)$.

In the $\mathrm{VV}$ ECMO subgroup, 0/17 $\mathrm{ECHO}+$ patients required cannula repositioning, while $4 / 20(25 \%)$ ECHO- patients required cannula repositioning ( $p=0.1$, Table 2$)$. In the VA ECMO subgroup, 2/9 (22\%) ECMO+ patients required cannula repositioning, while 2/43 (4.6\%) ECHO- patients required repositioning $(\mathrm{p}=0.07)$. 


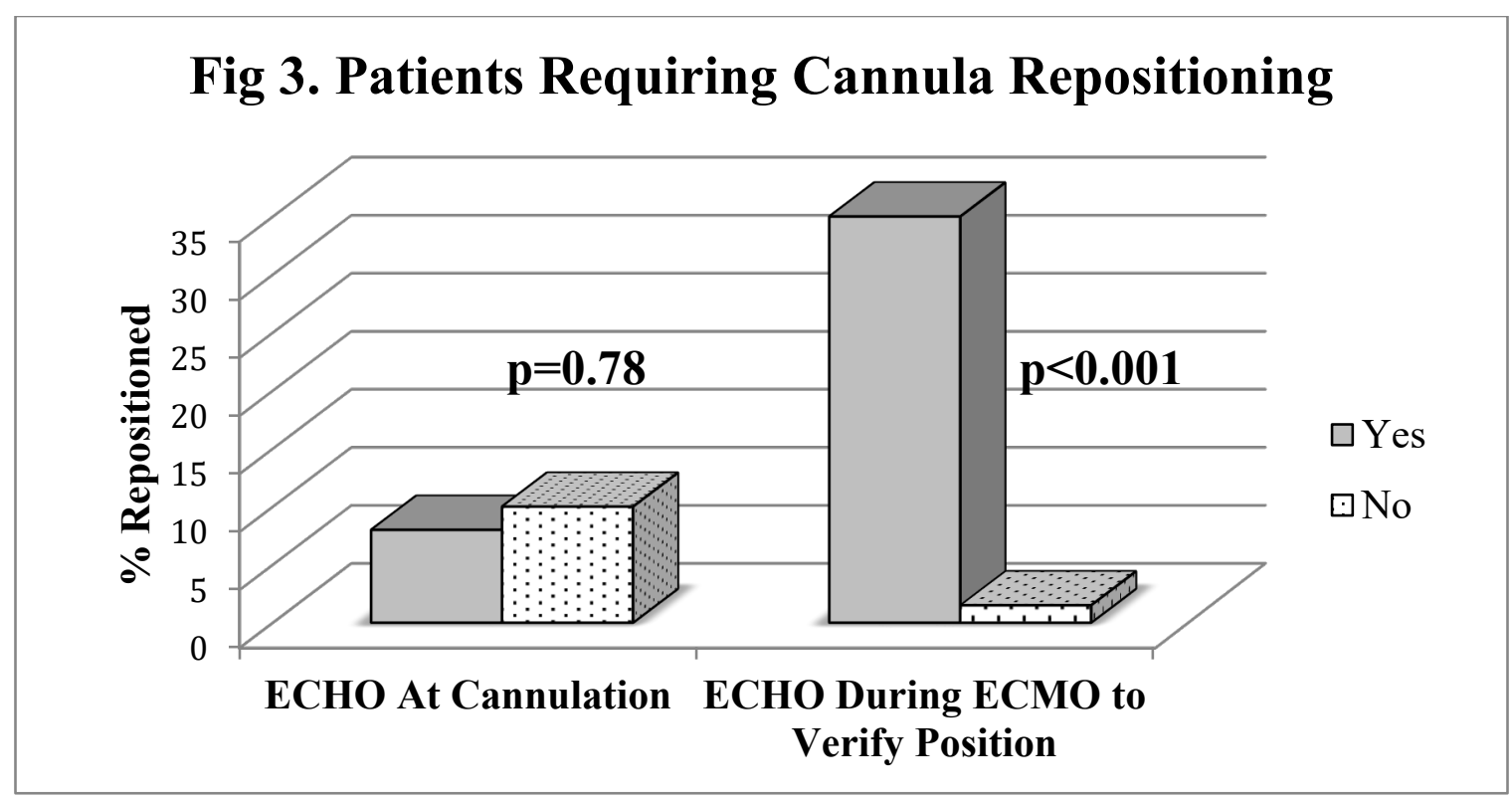

\begin{tabular}{|c|c|c|c|}
\hline Mode of Cannulation & ECHO (+) & ECHO (-) & P-Value \\
\hline VV & $0 / 17$ & $4 / 20$ & 0.10 \\
\hline VA & $2 / 9$ & $2 / 43$ & 0.13 \\
\hline
\end{tabular}

Table 2. Cannula Repositioning Separated by Mode of Cannulation

\section{Complications}

Each group had one major complication. In the ECHO+ group, there was an atrial guide wire perforation during a percutaneous VV cannulation. Fluoroscopy was not used in that procedure. In the ECHO- group, there was an atrial cannula perforation during the ECMO run after initial cannulation. This was also in the setting of a VV cannula. There was no significant difference in any minor complication when comparing $\mathrm{ECHO}+$ and $\mathrm{ECHO}-$ groups. Additionally, repositioning procedures resulted in no additional complications. Cannula site bleeding occurred in 37\% (3/8) of patients requiring cannula repositioning, and it occurred in $27 \%(22 / 81)$ of patients who did not require repositioning $(\mathrm{p}=0.53)$.

\section{Discussion}

Despite ECMO's use for over four decades, there are no evidence-based guidelines for the proper method of evaluating ECMO cannula position during cannulation, with most cannulators traditionally obtaining chest X-rays after cannula placement. Nevertheless, in a 1994 review of complications reported to the extracorporeal life support organization registry by Zwischenberger et al., cannula malposition occurred in $9 \%$ of all ECMO cannulations. ${ }^{5}$ Abnormal sites include against the interatrial septum, through a patent foramen ovale and into the left atrium, in the coronary sinus, and across the tricuspid valve or subvalvular apparatus, with venous cannulae being more likely to be incorrectly 
positioned than arterial cannulae. In addition, malpositioning may result in cardiac or vascular injury in addition to inadequate flows. A malpositioned cannula requires manipulation or reoperation to achieve the appropriate location for adequate flow and ECMO support. In turn, this places patients at risks for infections, bleeding, or cardiovascular injury, in addition to the added healthcare costs of re-intervention. Only more recently has data been presented in the literature to recommend ECHO or fluoroscopy during cannulation to avoid some of these issues. ${ }^{2-4,6}$

This study set out to assess whether the use of ECHO guidance during neonatal and pediatric jugular cannulation for ECMO reduced the need for cannula repositioning or decreased morbidity. In designing the protocol reported in this study, our goal was to perform ECHO on all ECMO patients during cannulation after initiation of the protocol. However, the results summarized in Table 1 and Figure 2 show a clear bias in our patient groups. The $\mathrm{ECHO}+$ group included a higher percentage of pediatric, respiratory, and VV patients, while the ECHO- group included more neonatal, cardiac/CDH, and VA patients. This result was interesting but not wholly unexpected. The OriGen dual-lumen cannula requires precise cannula tip placement for optimal flow and to prevent cardiovascular injury, whereas single lumen jugular venous cannula tip location in VA ECMO is generally less rigorous to attain proper support. In the end, individual surgeons chose whether or not to have an ECHO at cannulation. Perhaps they made their decisions based on comfort with the cannulation procedure or based on this element of precision. Further, most patients go on VV ECMO for respiratory failure, and it has been shown that patients undergoing VV cannulation have a higher likelihood of being older, as seen by Zahraa, et al. ${ }^{7}$

Our finding that there was no difference in cannula repositioning procedures required between the two groups conflicted with our initial hypothesis that ECHO would result in less repositioning procedures, as suggested by previous studies ${ }^{2-4}$. Cannula repositioning rates of 8 and 10 percent, while low in both groups, may have been affected by the small patient population in our study as compared to previously published work. Ours is the first study to compare repositioning procedures between the VV and VA populations. Of note, there were no cannula repositioning procedures required in our ECHO+ VV patients, as compared to $20 \%$ of the ECHO- VV group. This trended towards significance and shows an advantage of the use of ECHO during VV cannulation. To the contrary, the trend towards more repositioning in the $\mathrm{ECHO}+\mathrm{VA}$ patients shows no benefit of $\mathrm{ECHO}$ in that population.

We were also interested in whether the ECHO protocol would result in decreased resource utilization at our institution. As noted above, there were similar operating room resources used in repositioning procedures. Surprisingly, we found that the ECHO+ group underwent more ECHO studies per patient than the ECHO- group during the ECMO runs after initial cannulation. This could be due to the higher proportion of VV patients in the ECHO+ group requiring more $\mathrm{ECHO}$ studies for precise localization of the cannula tip. Alternatively, it could be due to more ECHO studies being ordered by the ICU providers in the later time period of the study, correlating with more ECHO+ patients. By encouraging the use of ECHO at cannulation, did more physicians begin to not only use it at cannulation, but also more freely during the respective ECMO runs to verify placement? 
With the retrospective nature of this study and sheer number of physicians caring for these patients, these decision points are difficult to decipher. A future study might prospectively follow a controlled group of patients with a rigid care protocol or small number of physicians making these decisions.

There were no significant differences in major or minor complications in both groups. However, it is important to note that ECHO did not prevent atrial perforation from a guidewire during a percutaneous VV cannulation in the absence of fluoroscopy, and even one atrial perforation is one too many. Thus, other adjuncts such as fluoroscopy should be considered in percutaneous cannulation. ${ }^{6}$ Unfortunately, these complications are not uncommon. In a retrospective institutional analysis, Rupprecht et al. have shown that one of the major complications of percutaneous ECMO is cannulation itself, with an $8.8 \%$ risk of vessel puncture and a $5.7 \%$ risk of significant blood loss. ${ }^{8}$ Additionally, bleeding complications on ECMO are common, and a high incidence of cannula site bleeding can be attributed to systemic anticoagulation, circuit-induced coagulopathy, and systemic disease processes. ${ }^{9}$ We found no difference in cannula site cervical bleeding between the two groups, and importantly, we found no effect of cannula repositioning on bleeding complications.

\section{Conclusion}

There are currently no guidelines that have been developed from large data sets with respect to the optimal imaging modality to help guide ECMO cannulation. This study shows that the implementation of an institutional protocol to encourage ECHO use during jugular cannulation for neonatal and pediatric ECMO did not result in significantly less repositioning procedures, complications, or resource utilization. However, ECHO may still be a useful adjunct for surgeons during difficult cannulations and for precise placement of dual-lumen VV cannulae. Further studies should prospectively follow patients in a protocolized care model to better estimate the effect of protocol change to help form better guidelines for clinicians when placing patients on ECMO support.

\section{References}

1. Hirose H, Yamane K, Marhefka G, Cavarocchi N. Right ventricular rupture and tamponade caused by malposition of the Avalon cannula for venovenous extracorporeal membrane oxygenation. J Cardiothorac Surg. 2012;7:36.

2. Irish MS, O'Toole SJ, Kapur P, Bambini DA, Azizkhan RG, Allen JE, Caty MG, Gilbert JC, Steinhorn RH, Glick PL. Cervical ECMO cannula placement in infants and children: recommendations for assessment of adequate positioning and function. J Pediatr Surg. 1998;33(6):929-31.

3. Kuenzler KA, Arthur LG, Burchard AE, Lawless ST, Wolfson PJ, Murphy SG. Intraoperative ultrasound reduces ECMO catheter malposition requiring surgical correction. J Pediatr Surg. 2002;37(5):691-4. 
4. Thomas TH, Price R, Ramaciotti C, Thompson M, Megison S, Lemler MS. Echocardiography, not chest radiography, for evaluation of cannula placement during pediatric extracorporeal membrane oxygenation. Pediatr Crit Care Med. 2009;10(1):56-9.

5. Zwischenberger JB, Nguyen TT, Upp JR Jr, Bush PE, Cox CS Jr, Delosh T, Broemling L. Complications of neonatal extracorporeal membrane oxygenation. Collective experience from the extracorporeal life support organization. J Thorac Cardiovasc Surg. 1994:107(3):838-48.

6. Teman NR, Haft JW, Napolitano LM. Optimal endovascular methods for placement of dual-lumen cannulae for venovenous extracorporeal membrane oxygenation. ASAIO J. 2013 Jul-Aug;59(4):442-7.

7. Zahraa JN, Moler FW, Annich GM, Maxvold NJ, Bartlett RH, Custer JR. Venovenous versus venoarterial extracorporeal life support for pediatric respiratory failure: are there differences in survival and acute complications? Crit Care Med. 2000;28(2):521-5.

8. Rupprecht L, Lunz D, Philipp A, Lubnow M, Schmid C. Pitfalls in percutaneous ECMO cannulation. Heart, Lung and Vessels. 2015;7(4):320-326

9. Thomas J, Kostousov V, Teruya J. Bleeding and thrombotic complications in the use of extracorporeal membrane oxygenation. Semin Thromb Hemost. 2018:44(1):20-29. 